\title{
Exchange Coupling Effects in Naturally Oxidised Ultrathin Iron Film Acta Physica Polonica A 133, 601 (2018), ERRATUM
}

H. DAWCZAK-DęBICKi ${ }^{a, b}$, A. MARczyńska ${ }^{a}$, A. Rogowska $^{a, c}$, M. WACHOWIAK $^{a, c}$, M. Nowicki ${ }^{c}$, S. PACANOWSKI ${ }^{a}$, B. JabŁoński ${ }^{a, c}$, R. CzAJKA ${ }^{c}$ And L. Smardz ${ }^{a, *}$

${ }^{a}$ Institute of Molecular Physics, Polish Academy of Sciences, M. Smoluchowskiego 17, 60-179 Poznań, Poland

${ }^{b}$ Faculty of Physics, Adam Mickiewicz University, Umultowska 85, 61-614 Poznań, Poland

${ }^{c}$ Faculty of Technical Physics, Poznań University of Technology, Piotrowo 3, 60-965 Poznań, Poland

Oxidation process of Fe films under atmospheric conditions is depth limited such that an oxide covering layer with a well-defined thickness is formed by which the underlying metal is prevented from further oxidation. Iron thin film with an initial thickness $d_{i}=4 \mathrm{~nm}$ was deposited onto $1.6 \mathrm{~nm}-\mathrm{V}(110)$ buffer layer using UHV magnetron sputtering. The planar growth of Fe oxides was revealed by atomic force microscopy. X-ray photoelectron spectroscopy studies performed after 250 days of oxidation revealed formation of a hematite $\left(\alpha-\mathrm{Fe}_{2} \mathrm{O}_{3}\right)$ ultrathin film on the metallic rest of iron. Furthermore, low temperature magnetic measurements of the oxidised Fe ultrathin film revealed an exchange anisotropy which is imposed to the metallic rest. As a result, we have observed at low temperatures a shift and broadening of the hysteresis loops due to the exchange interaction at the metal-oxide interface.

DOI: 10.12693 /APhysPolA.136.571

PACS/topics: 75.70.-i; 75.70.Kw

This article was originally published on March 2018 with incorrect Fig. 3a. The correct version of Fig. 3a is included below.



The authors apologize for this error.

*corresponding author; e-mail: smardz@ifmpan.poznan.pl 